\title{
Numerical simulation for water exit of an initially fully-submerged buoyant spheroid in an axisymmetric flow
}

\author{
B.Y. $\mathrm{Ni}^{1}$, G.X. Wu ${ }^{*^{1,2}}$ \\ 1. College of Shipbuilding Engineering, Harbin Engineering University, Harbin 150001, P. R. China; \\ 2. Permanent address: Department of Mechanical Engineering, University College London, London
}

WCIE 7JE, UK;

\begin{abstract}
:
Free water exit of an initially fully-submerged buoyant spheroid in an axisymmetric flow, which is driven by the difference between vertical fluid force and gravity, is investigated. The fluid is assumed to be incompressible and inviscid, and the flow to be irrotational. The velocity potential theory is adopted together with the fully nonlinear boundary conditions on the free surface. Surface tension has been neglected and the pressure is taken as constant on the free surface. The acceleration of the body at each time step is obtained as the part of the solution. Its nonlinear mutual dependence with the fluid force is decoupled through the auxiliary function method. The free-surface breakup by the body penetration and the water detachment from the body are treated through numerical conditions. Slender body theory based on the zero potential assumption on the undisturbed flat free surface is adopted, through which a condition for full water exit of a spheroid is obtained. Comparison is made between the results from the slender body theory and from the fully nonlinear theory through the boundary-element method, and good agreement is found when the spheroid is slender. Extensive case studies are undertaken to investigate the effects of the body density, dimensions and the initial submergence.
\end{abstract}

Key words: Water exit; Submerged buoyant body; Free motion; Critical body density

\section{Introduction}


Water entry and exit have been the topics of extensive interests in hydrodynamics because of their wide range of applications in naval architecture, ocean engineering and costal engineering, etc. There has been a large body of the work on water entry (Scolan \& Korobkin (2001), Baarholm \& Faltinsen (2004), Moore et al. (2012) and Wu \& Sun (2014)). Relative to water entry, there has been far less work on water exit. Part of the reason is that instead of the increase of fluid loads, or forces, during water entry, they decrease usually during water exit, during which the wetted surface decreases with time and the liquid and body may detach from each other eventually. The fluid loading in many cases is not as excessive as that in water entry problems. Another reason is that it is difficult to use the Wagner theory, which uses a linear free surface boundary condition with a correction for the waterline and is commonly used in the water entry, in the water exit problem. In other words, it is not easy to reverse the process of water entry to water exit directly in the mathematical method. However, water exit is also an important engineering problem. In fact, for a ship in rough seas, its bow will repeatedly emerge from water and then hit water surface, which forms a continuous water exit/entry process. In the sport, measurement has been made for the pressure/acceleration change during water exit of the head of a breaststroke swimmer (Pansiot et al., 2010).

There are two major difficulties in water exit problem, related to free-surface breakup and water detachment from the body (Ni et al., 2015). Early work on water exit was usually based on the assumption of the flat free surface. Therefore when the lowest point of the body was above the undisturbed water level, the body was thought to be fully exited. Moran (1963) developed a second-order theory for a slender body of resolution by expanding the boundary condition based on the ratio of the transverse and longitudinal dimensions of the body. He then considered an image solution for a constant-strength point source moving towards a free boundary (Moran 1964) and further gave a review on the problem (Moran 1965). Recently Tassin et al. (2013) studied the water exit problem of a body with time-varying shape, which entered water initially and then reversed its vertical speed at later stage. The free surface was elevated based on the Wagner theory used during water entry stage. Korobkin (2013) studied the water exit of two dimensional (2D) 
and axisymmetric bodies, which were initially partially submerged and suddenly started to move upwards at a constant acceleration. Boundary conditions on the liquid surface were linearized and imposed on its undisturbed position. The wetted surface of the body was simplified by using the so-called 'equivalent flat-plate approximation'. This was extended by Korobkin et al. (2014) for a prescribed and varying acceleration, and extended by Khabakhpasheva et al. (2015) for the problem similar to that considered by Tassin et al. (2013).

For the problem with the deforming free surface caused by the body motion, Greenhow \& Moyo (1997) considered a 2D circular cylinder by using the boundary-element method (BEM) based on complex velocity potential. The free-surface shape was tracked through the fully nonlinear boundary condition. They simulated the fluid flow during the rise of the cylinder at a given vertical velocity. The results at initial stage were compared with those from small-time expansion solution by Tyvand \& Miloh (1995) and simulation was terminated before the breakup of the free surface or body piercing. Liju et al. (2001) considered water exit of an axisymmetric body at different Froude number using BEM. Numerical result was compared with their experimental data from a model test in small scale. The simulation was also terminated before body piercing.

Rajavaheinthan \& Greenhow (2015) studied water exit of 2D bodies at constant acceleration using BEM with the fully nonlinear free-surface boundary condition. The body was initially floating on the free surface and therefore it was already beyond the free-surface breakup stage. They calculated the free-surface deformation and terminated the simulation before fully water exit. A very recent work by $\mathrm{Ni}$ et al (2015) considered the entire process of the water exit of an axisymmetric body. The body was initially fully submerged and was given a vertical constant speed. The deformation of the free surface was followed based on the fully nonlinear theory. As the body continued to move up, it would first pierce the free surface and would fully exit water later. The whole process was simulated through the axisymmetric BEM with time stepping technique (Longuet-Higgins \& Cokelet 1976).

In all these cases above, the body speed is prescribed, whether it is constant or varying with time. In the present work, we shall solve the complete problem of water exit of an initially fully 
submerged buoyant body including determination of the free motion. When the body is lighter than liquid, it will move up and pierce the free surface. When the density is sufficiently small, the body is expected to move further up and exit water fully. This phenomenon is also known as 'pop up' or 'pop off', which was studied based on experiments by Bourrier et al. (1984) and by Truscott et al. (2016). During the simulation of this process, the difficulty associated with the mutual dependence of the body acceleration and the fluid force will be decoupled by the auxiliary function method (Wu \& Eatock Taylor, 2003). The free-surface breakup and liquid/body detachment will be solved based on the method described in Ni et al. (2015). A condition for the density below which the body will exit water fully will be derived based on the slender body theory. Extensive case studies based on spheroids will be undertaken for the effects of body density, dimensions and submergence.

\section{Mathematical model and numerical procedure}

\subsection{Mathematical model}

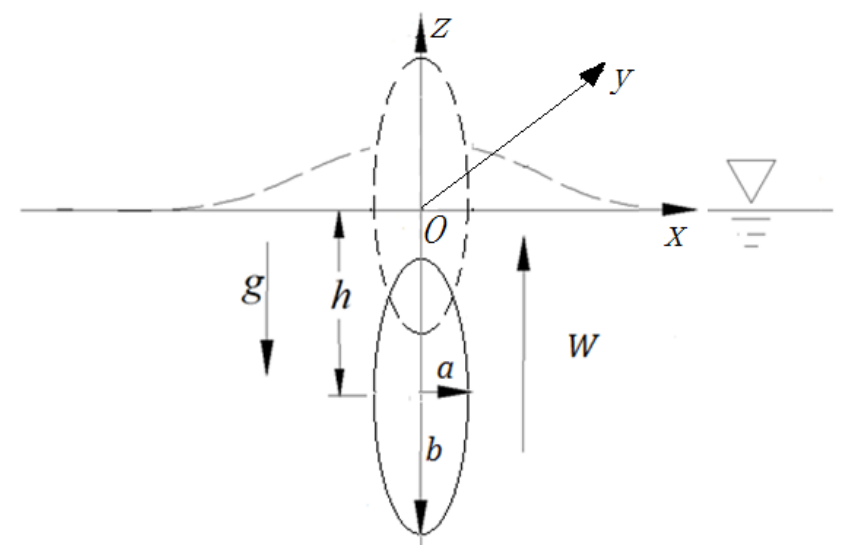

Fig. 1. Sketch of the problem with initial position and velocity of the buoyant body as well as the Cartesian coordinate system $O-x y z$

We consider the vertical water exit of a buoyant body. Without loss of generality, the body is a prolate ellipsoid, oriented with its axis coinciding with the vertical $z$-axis of coordinates, and it has two semi-axes, $b$ and $a$, where $0<a \leq b$, as shown in Fig 1 . Initially the water surface is calm and the spheroid is fully-submerged with the depth of submergence as $h$. A Cartesian 
coordinate system $O-x y z$ as well as a cylindrical coordinate system $O-r \theta z$ are defined, in which the origin $O$ is located on the undisturbed free surface and $z$ points vertically upwards and passes through the major axis of the body. When the body motion is along the $z$ axis, the flow is then axisymmetric. The fluid is assumed to be incompressible and inviscid, and the flow to be irrotational. Thus the fluid flow can be described in terms of velocity potential $\Phi$, which satisfies the Laplace's equation:

$$
\nabla^{2} \Phi=0
$$

in the fluid domain. The impermeable boundary condition on the wetted part of the rigid body surface $s_{b}$ is given by

$$
\frac{\partial \Phi}{\partial n}=[W(t) \boldsymbol{k}] \cdot \boldsymbol{n}=W(t) \cdot n_{z},
$$

where $W(t)$ is the vertical velocity of the body, which is a function of time and needs to be found at each time step, $\boldsymbol{k}$ is the unit vector along $z$ axis, $\boldsymbol{n}=\left(n_{r}, n_{z}\right)$ is the normal vector of the body surface pointing out of the fluid domain and $n_{z}$ is its $z$ component. On the free surface $s_{f}$, the fully nonlinear kinematic and dynamic boundary conditions can be written below in the Lagrangian framework:

$$
\begin{gathered}
\frac{D r}{D t}=\frac{\partial \Phi}{\partial r}, \quad \frac{D z}{D t}=\frac{\partial \Phi}{\partial z}, \\
\frac{D \Phi}{D t}=\frac{1}{2}|\nabla \Phi|^{2}-g z,
\end{gathered}
$$

where $D / D t$ is the substantial derivative following a fluid particle, $g$ is the acceleration due to gravity. Bernoulli equation and constant pressure on the free surface have been used in Eq.(4). The boundary condition at infinity $s_{\infty}$ is based on the assumption that the fluid there is undisturbed. We have

$$
\Phi=O\left(\frac{1}{r^{2}+z^{2}}\right) . \quad \sqrt{r^{2}+z^{2}} \rightarrow \infty
$$

As both the body and the water surface are initially stationary, the initial conditions can be given as $W(0)=0$ for the body and $\Phi=0$ on the flat free surface. Eq. (1) can then be solved together 
with the boundary conditions Eqs.(2)-(5) and initial conditions as an initial-boundary-value problem (IBVP).

\subsection{Boundary-element method}

By using the Green's third identity with the Green function $G$, Laplace's equation can be converted into the following boundary integral equation:

$$
\varepsilon(\boldsymbol{p}) \Phi(\boldsymbol{p})=\iint_{s}\left(\frac{\partial \Phi(\boldsymbol{q})}{\partial n_{q}} G(\boldsymbol{p}, \boldsymbol{q})-\Phi(\boldsymbol{q}) \frac{\partial}{\partial n_{q}} G(\boldsymbol{p}, \boldsymbol{q})\right) d s,
$$

where $s$ is the domain boundary including $s_{b}, s_{f}$ and $s_{\infty}$. In the equation $p$ is the field point, $\boldsymbol{q}$ is the integral (or the source) point on the boundary and $\varepsilon(\boldsymbol{p})$ is the solid angle when observing the flow field at point $\boldsymbol{p}$. The Green function in the three-dimensional domain is $G(\boldsymbol{p}, \boldsymbol{q})=1 /|\boldsymbol{R}-\boldsymbol{r}|$, where $\boldsymbol{R}=\left(R, Z, \theta_{2}\right)$ and $\boldsymbol{r}=\left(r, z, \theta_{1}\right)$ are the position vectors of $\boldsymbol{p}$ and $\boldsymbol{q}$ respectively.

The boundary-element method is adopted to solve Eq.(6). The detailed procedure was provided in Ni et al. (2015) and only a brief description is provided here as follows. The integration of $d s=r d \theta d l$ can be split into those with respect to $\theta$ and $r$ respectively, where $d l$ is an element of infinitesimal arclength of a meridian line drawn on the body. Because of the axisymmetry of the problem, the former can be performed explicitly. The remaining integral will be with respect to $r$ along a closed line $l=l_{b}+l_{f}+l_{\infty}$. This is then divided into many straight line elements and the linear shape function is used within each element ( $\mathrm{Ni}$ et al. 2013). The integration over $l_{\infty}$ can be replaced by that over a control surface $l_{c}$ at a large distance from the body, where $\partial \Phi / \partial n=0$ is imposed.

Nondimensionalisation is adopted to make the problem general. Different from that in $\mathrm{Ni}$ et al. (2015), the initial velocity of the body is zero and therefore it is not suitable to be used as one of 
the base parameters. Instead, nondimensionalisation is applied based on the semi major axis $b$, the acceleration $g$ due to gravity, and the density of the fluid $\rho$. Thus we use $\sqrt{b / g}$ for time, $\sqrt{g b}$ for the velocity, $\sqrt{g b^{3}}$ for the velocity potential and $\rho g b$ for pressure respectively. The nondimensionalized parameters will then be denoted by a bar. The boundary conditions on the body surface and the free surface can be rewritten as:

$$
\begin{gathered}
\frac{\partial \bar{\Phi}}{\partial n}=\bar{W} \cdot n_{z}, \\
\frac{D \bar{r}}{D \bar{t}}=\frac{\partial \bar{\Phi}}{\partial \bar{r}}, \quad \frac{D \bar{z}}{D \bar{t}}=\frac{\partial \bar{\Phi}}{\partial \bar{z}}, \\
\frac{D \bar{\Phi}}{D \bar{t}}=\frac{1}{2}|\nabla \bar{\Phi}|^{2}-\bar{z},
\end{gathered}
$$

where $\bar{W}=W / \sqrt{g b}$ is the dimensionless velocity of the body which varies with time. In addition, we define $\lambda=h / b$ as the initial submergence parameter.

Time stepping method will be adopted to solve this boundary-initial-value problem. Without loss of generality, the initial conditions can be set as $\bar{W}(0)=0$ for an initially static body and $\bar{\Phi}=0$ on the initially calm free surface. As a result, the buoyant body will rise just relying on the fluid force. As in Ni et al. (2015), the Euler method for differential equations is adopted in time-stepping to update both the body velocity/position and the shape of and the velocity potential on the free surface. The choice of the time step is based on the velocity and acceleration of fluid particle, or:

$$
\begin{gathered}
\Delta \overline{t_{1}}=\min \{C \cdot \Delta \bar{l} /|\nabla \bar{\Phi}|\}, \\
\Delta \bar{t}_{2}=D / \max \left\{\frac{1}{2}|\nabla \bar{\Phi}|^{2}-\bar{z}\right\}, \\
\Delta \bar{t}=\min \left\{\Delta \bar{t}_{1}, \Delta \bar{t}_{2}\right\},
\end{gathered}
$$

where $\Delta \bar{l}$ and $\nabla \bar{\Phi}$ are respectively the length of a local element and the corresponding fluid particle velocity at the element, and $C$ and $D$ are constants. Through Eqs.(10), the changes of both 
the position of a fluid particle and the velocity potential are confined to ensure stability and accuracy. Based on the discussion in Ni et al.(2015), we adopt $C=1 / 120$ and $D=1 / 2400$ in this paper.

During water exit, the body will break up the free surface when its front emerges from water, and detach from the free surface when its back departs from water. The breakup and detachment of the free surface must be carefully treated in numerical modelling. Following the procedure described in Ni et al. (2015), it is assumed that the free surface will break up when the thickness of the water layer right above the top of the body is smaller than a critical distance $\Delta \overline{l_{c}}$. Before the back of the body detaches from the water, a water column is usually seen to be attached to the body surface. It is assumed when the radius of the water column is smaller than $\Delta \bar{l}_{c}$, body/liquid detachment will occur in the numerical simulation at the next time step. Ni et al. (2015) have undertaken extensive numerical investigations and found that the results of interest are not sensitive to the choice of $\Delta \bar{l}_{c}$ when it is sufficiently small. It is taken as $10 \%$ of the element size on the body in the present work.

\subsection{Decoupling of the motion of the body and fluid flow}

For a buoyant submerged body driven into motion by the difference between the fluid force and the weight, $W(t)$ in Eq.(2) is unknown and needs to be found from integrating the acceleration $d W / d t$ with respect to time. However $d W / d t$ is directly related to fluid force which further depends on the body motion. One way to decouple the nonlinear mutual dependence is to use the auxiliary function method (Wu \& Eatock Taylor, 2003), which can be summarised below.

By using Newton's second Law, the body motion equation is

$$
\bar{m} \frac{d \bar{W}}{d \bar{t}}=\bar{F}+\bar{F}_{e}
$$

in which the mass of the body $\bar{m}=\bar{\rho}_{B} \bar{V}_{B}=\bar{\rho}_{B} 4 \pi \bar{a}^{2} / 3$ with constant body density $\bar{\rho}_{B}, \bar{F}_{e}$ is 
the external force exerted on the body, which is $\bar{F}_{e}=-\bar{m}$ when only the gravity is considered.

$\bar{F}$ is the fluid force and can be obtained by integrating the fluid pressure obtained from the Bernoulli equation over the wetted body surface:

$$
\bar{F}=\bar{F}_{z}=\iint_{\bar{s}_{b}} \bar{P} \cdot n_{z} d \bar{s}=-\iint_{\bar{s}_{b}}\left(\frac{\partial \bar{\Phi}}{\partial \bar{t}}+\frac{1}{2}|\nabla \bar{\Phi}|^{2}+\bar{z}\right) \cdot n_{z} d \bar{s} .
$$

The term $\partial \bar{\Phi} / \partial \bar{t}$ on the right hand side of Eq.(12) is problematic in direct numerical calculation through time-difference method . We follow the method proposed by Wu \& Eatock Taylor (2003). $\partial \bar{\Phi} / \partial \bar{t}$ can be seen as a harmonic function, which satisfies Laplace's equation,

$$
\nabla^{2} \frac{\partial \bar{\Phi}}{\partial \bar{t}}=0
$$

The boundary condition for $\partial \bar{\Phi} / \partial \bar{t}$ on the free surface is

$$
\frac{\partial \bar{\Phi}}{\partial \bar{t}}=-\frac{1}{2}|\nabla \bar{\Phi}|^{2}-\bar{z}
$$

A relation valid on the body surface can be written as (Wu, 1998):

$$
\frac{\partial(\partial \bar{\Phi} / \partial \bar{t})}{\partial n}=\frac{d \bar{W}}{d \bar{t}} n_{z}-\bar{W} \frac{\partial(\partial \bar{\Phi} / \partial \bar{z})}{\partial n} .
$$

Here we introduce two auxiliary functions $\chi_{1}(\bar{x}, \bar{y}, \bar{z}, \bar{t})$ and $\chi_{2}(\bar{x}, \bar{y}, \bar{z}, \bar{t})$ (Wu et al., 2004) and let

$$
\frac{\partial \bar{\Phi}}{\partial \bar{t}}=\frac{d \bar{W}}{d \bar{t}} \chi_{1}+\chi_{2}-\bar{W} \frac{\partial \bar{\Phi}}{\partial \bar{z}}
$$

$\chi_{1}$ and $\chi_{2}$ both satisfy the Laplace's equation in the fluid domain and the following boundary conditions, 


$$
\left\{\begin{array} { l } 
{ \frac { \partial \chi _ { 1 } } { \partial n } = n _ { z } \quad \text { on } \quad \overline { s } _ { b } } \\
{ \chi _ { 1 } = 0 \quad \text { on } \quad \overline { s } _ { f } } \\
{ \frac { \partial \chi _ { 1 } } { \partial n } = 0 \quad \text { on } \quad \overline { s } _ { c } }
\end{array} \quad \text { and } \quad \left\{\begin{array}{l}
\frac{\partial \chi_{2}}{\partial n}=0 \quad \text { on } \bar{s}_{b} \\
\chi_{2}=-\frac{1}{2}|\nabla \bar{\Phi}|^{2}-\bar{z}+\bar{W} \frac{\partial \bar{\Phi}}{\partial \bar{z}} \quad \text { on } \quad \bar{s}_{f} \\
\frac{\partial \chi_{2}}{\partial n}=0 \quad \text { on } \quad \bar{s}_{c}
\end{array}\right.\right.
$$

The problems for $\chi_{1}$ and $\chi_{2}$ can be easily solved by a similar procedure used for the velocity potential $\bar{\Phi}$. Subsequently the body motion equation can be found by substituting Eqs.(16) and (12) into Eq.(11). We have

$$
\bar{m} \frac{d \bar{W}}{d \bar{t}}=-\iint_{\bar{s}_{b}}\left(\frac{d \bar{W}}{d \bar{t}} \chi_{1}+\chi_{2}-\bar{W} \frac{\partial \bar{\Phi}}{\partial \bar{z}}+\frac{1}{2}|\nabla \bar{\Phi}|^{2}+\bar{z}\right) n_{z} d \bar{s}+\bar{F}_{e},
$$

which can also be written as

$$
\left(\bar{m}+\bar{m}_{a}\right) \frac{d \bar{W}}{d \bar{t}}=-\iint_{\bar{s}_{b}}\left(\chi_{2}-\bar{W} \frac{\partial \bar{\Phi}}{\partial \bar{z}}+\frac{1}{2}|\nabla \bar{\Phi}|^{2}+\bar{z}\right) n_{z} d \bar{s}+\bar{F}_{e},
$$

where

$$
\bar{m}_{a}=\iint_{\bar{s}_{b}} \chi_{1} n_{z} d \bar{s}
$$

which is an added mass (Wu \& Eatcok Taylor 2003). After $\chi_{1}$ and $\chi_{2}$ solved, $d \bar{W} / d \bar{t}$ can be easily obtained by solving Eq. (19). As a result, the fluid force on the body is:

$$
\bar{F}=\bar{m} \frac{d \bar{W}}{d \bar{t}}-\bar{F}_{e}
$$

\subsection{Slender body theory}

For a small value of the slenderness $a / b$ such that $0<a / b<<1$, we may adopt the slender body theory. The force $\bar{F}$ in Eq.(11) can be decomposed into the hydrodynamic one $\bar{F}_{d}$ and the hydrostatic one $\bar{F}_{s}$ :

$$
\bar{m} \frac{d \bar{W}}{d \bar{t}}=\bar{F}_{d}+\bar{F}_{s}-\bar{m}
$$


When the body is slender, the free surface can be assumed to be undisturbed, and $\bar{\Phi}=0$ on $\bar{z}=0$, Within this framework the hydrodynamic force can be written as (Wu 1998)

$$
\bar{F}_{d}=-\frac{1}{2} \frac{d \bar{m}_{a}}{d \bar{t}} \bar{W}-\bar{m}_{a} \frac{d \bar{W}}{d \bar{t}}
$$

where the added mass

$$
\bar{m}_{a}=\iint_{\bar{s}_{b}} \psi \frac{\partial \psi}{\partial n} d \bar{s}=\iint_{\bar{s}_{b}} \psi n_{z} d \bar{s}
$$

with $\psi=\bar{\Phi} / \bar{W}$. Here $\bar{m}_{a}$ is slightly different from that in Eq.(20), as $\psi$ here satisfies $\psi=0$ on $\bar{z}=0$ while $\chi_{1}$ in Eq.(20) satisfies $\chi_{1}=0$ on $\bar{z}=\eta$. The hydrostatic force can be written as

$$
\bar{F}_{s}=-\iint_{\bar{s}_{b}} \bar{z} n_{z} d \bar{s}=\bar{V}_{d},
$$

where $\bar{V}_{d}$ is the volume of the body below the flat free surface, which varies with time when the body has pierced the free surface.

Substituting Eqs.(23) and (25) into (22), we have

$$
\left(\bar{m}+\bar{m}_{a}\right) \frac{d \bar{W}}{d \bar{t}}+\frac{1}{2} \frac{d \bar{m}_{a}}{d \bar{t}} \bar{W}=\left(\bar{F}_{s}-\bar{m}\right) .
$$

For a prolate ellipsoid, one has

$$
\bar{V}_{d} / \bar{V}_{B}=\left\{\begin{array}{ll}
1 & \bar{z}_{c}<-1 \\
\frac{1}{2}-\frac{3}{4} \bar{z}_{c}+\frac{1}{4} \bar{z}_{c}^{3} & -1 \leq \bar{z}_{c} \leq 1
\end{array},\right.
$$

where $\bar{z}_{c}$ is the vertical coordinate of the centre of the body and $\bar{V}_{B}$ is the volume of the full prolate ellipsoid defined after Eq.(11).

Based on the slender body theory (Mackie, 1962, Newman, 1977), $\psi$ in Eq.(24) can be written as

$$
\psi(\bar{z}, \bar{r})=-\frac{1}{4 \pi} \int_{\bar{z}_{c}-1}^{-\bar{z}_{c}+1} \frac{\bar{S}^{\prime}(\xi) d \xi}{\left[(\bar{z}-\xi)^{2}+\bar{r}^{2}\right]^{1 / 2}},
$$


where $\bar{S}(\xi)$ is the cross-sectional area of the body at $\bar{z}=\xi$ and $\bar{S}^{\prime}(\xi)$ is its derivative. For the case of a prolate ellipsoid,

$$
\bar{S}(\xi)=\left\{\begin{array}{ll}
\pi \bar{a}^{2}\left[1-\left(\xi-\bar{z}_{c}\right)^{2}\right] & \left(\bar{z}_{c}-1<\xi<0\right) \\
\pi \bar{a}^{2}\left[1-\left(\xi+\bar{z}_{c}\right)^{2}\right] & \left(0<\xi<1-\bar{z}_{c}\right)
\end{array} .\right.
$$

and hence

$$
\bar{S}^{\prime}(\xi)= \begin{cases}-2 \pi \bar{a}^{2}\left(\xi-\bar{z}_{c}\right) & \left(\bar{z}_{c}-1<\xi<0\right) \\ -2 \pi \bar{a}^{2}\left(\xi+\bar{z}_{c}\right) & \left(0<\xi<1-\bar{z}_{c}\right)\end{cases}
$$

Substituting $\bar{S}^{\prime}(\xi)$ into Eq.(28), after integration we can obtain:

$$
\begin{aligned}
\psi(\bar{z}, \bar{r})= & \frac{\bar{a}^{2}}{2}\left\{-\sqrt{\left(\bar{z}_{c}-1-\bar{z}\right)^{2}+\bar{r}^{2}}+\left(\bar{z}-\bar{z}_{c}\right) \ln \frac{-\bar{z}+\sqrt{\bar{z}^{2}+\bar{r}^{2}}}{\bar{z}_{c}-1-\bar{z}+\sqrt{\left(\bar{z}_{c}-1-\bar{z}\right)^{2}+\bar{r}^{2}}},\right. \\
& \left.+\sqrt{\left(1-\bar{z}_{c}-\bar{z}\right)^{2}+\bar{r}^{2}}+\left(\bar{z}+\bar{z}_{c}\right) \ln \frac{1-\bar{z}_{c}-\bar{z}+\sqrt{\left(1-\bar{z}_{c}-\bar{z}\right)^{2}+\bar{r}^{2}}}{-\bar{z}+\sqrt{\bar{z}^{2}+\bar{r}^{2}}}\right\}
\end{aligned}
$$

where $\bar{r}=\bar{a} \sqrt{1-\left(\bar{z}-\bar{z}_{c}\right)^{2}}$ when the potential is considered on the body surface.

For the axisymmetric problem Eq.(24) can be converted into

$$
\bar{m}_{a}=2 \pi \int_{\bar{l}_{b}} \psi n_{z} \bar{r} d \bar{l}=2 \pi \int_{\bar{z}_{c}-1}^{0} \frac{\psi n_{z} \bar{r}}{\sqrt{1-n_{z}^{2}}} d \bar{z}
$$

where $n_{z}=\frac{\bar{a}\left(\bar{z}-\bar{z}_{c}\right)}{\sqrt{1+\left(\bar{a}^{2}-1\right)\left(\bar{z}-\bar{z}_{c}\right)^{2}}}$. By substituting Eq.(29) into Eq.(30), one can obtain the added mass. Through Eq. (26) one has the acceleration of the body

$$
\frac{d \bar{W}}{d \bar{t}}=\left(\bar{F}_{s}-\bar{m}-\frac{1}{2} \frac{d \bar{m}_{a}}{d \bar{t}} \bar{W}\right) /\left(\bar{m}+\bar{m}_{a}\right)=\left(\bar{F}_{s}-\bar{m}-\frac{1}{2} \frac{d \bar{m}_{a}}{d \bar{z}_{c}} \bar{W}^{2}\right) /\left(\bar{m}+\bar{m}_{a}\right) .
$$

Defining $\Pi=\bar{W}^{2}$ and noticing

$$
\frac{d \bar{W}}{d \bar{t}}=\frac{d \bar{W}}{d \bar{z}_{c}} \bar{W}=\frac{1}{2} \frac{d \bar{W}^{2}}{d \bar{z}_{c}},
$$

we obtain a linear ordinary differential equation for $\Pi$,

$$
\frac{d \Pi}{d \bar{z}}=-\frac{d \bar{m}_{a} / d \bar{z}_{c}}{\bar{m}+\bar{m}_{a}} \Pi+2 \frac{\bar{F}_{s}-\bar{m}}{\bar{m}+\bar{m}_{a}} .
$$


This can be solved through the standard procedure, from which with the initial condition $\bar{W}(0)=0$, we have

$$
\bar{W}=\sqrt{\Pi}=\sqrt{\frac{2 \int_{-\lambda}^{\bar{z}_{c}}\left(\bar{F}_{s}-\bar{m}\right) d \bar{z}}{\bar{m}+\bar{m}_{a}}}=\sqrt{\frac{f\left(\bar{z}_{c}\right)}{\bar{\rho}_{B}+k_{s}}},
$$

where $f\left(\bar{z}_{c}\right)=\frac{1}{8} \bar{z}_{c}^{4}-\frac{3}{4} \bar{z}_{c}^{2}+\left(1-2 \bar{\rho}_{B}\right) \bar{z}_{c}+\left(2-2 \bar{\rho}_{B}\right) \lambda-\frac{3}{8}$ with $\lambda=h / b$ as defined in section 2.2, and $k_{s}=\bar{m}_{a} / \bar{V}_{B}$ is the added mass coefficient.

Substituting Eq.(34) into Eq.(31), we have

$$
\frac{d \bar{W}}{d \bar{t}}=\frac{\bar{F}_{s}-\bar{m}}{\bar{m}+\bar{m}_{a}}-\frac{\frac{d \bar{m}_{a}}{d \bar{z}_{c}} \int_{-\lambda}^{\bar{c}_{c}}\left(\bar{F}_{s}-\bar{m}\right) d \bar{z}}{\left(\bar{m}+\bar{m}_{a}\right)^{2}}=\frac{\frac{\bar{V}_{d}}{\bar{V}_{B}}-\bar{\rho}_{B}}{\bar{\rho}_{B}+k_{s}}-\frac{\frac{d k_{s}}{d \bar{z}_{c}} \frac{f\left(\bar{z}_{c}\right)}{2}}{\left(\bar{\rho}_{B}+k_{s}\right)^{2}} .
$$

In order to be sure that the body rises wholly out of the water (above the initial still water level), we must have $\bar{W} \geq 0$ when $\bar{z}_{c}=1$. This gives us a condition to find the critical density $\bar{\rho}_{B, c}$ of the body, below which the body will exit water fully. By substituting $\bar{W}=0$ and $\bar{z}_{c}=1$ into Eq.(34), one can calculate from $f(1)=0$ to obtain

$$
\bar{\rho}_{B, c}=\frac{\lambda}{1+\lambda} .
$$

Under this condition Eq.(35) gives $d \bar{W} / d \bar{t}=-1$. This in fact means that the acceleration equals to that due to gravity, which is expected as there is no fluid force at this moment. As $\lambda>1$ for an initially fully-submerged body, we have $1 / 2<\bar{\rho}_{B, c}<1$. When $\bar{\rho}_{B}<\bar{\rho}_{B, c}$, the body will exit water fully. When $\bar{\rho}_{B, c}<\bar{\rho}_{B}<1$, the body will exit water partially before it falls down. For the latter case, one can calculate the largest vertical coordinate $\bar{z}_{c}$ from $f\left(\bar{z}_{c}\right)=0$. 


\section{Numerical Results and Discussions}

\subsection{Convergence study and comparison study}

\subsubsection{Convergence study}

We first undertake the convergence study of a buoyant body with different meshes. The body has a small density $\bar{\rho}_{B}=0.2$ relative to the fluid, a dimensionless minor axis $\bar{a}=1 / 4$ and submergence parameter $\lambda=1.1$, which will burst out of the water totally based on the slender body theory in Eq.(36). The initial total number of elements on the body surface in the meridian plane is taken as $N_{b}=40$ and $N_{b}=60$ respectively. Elements of equal size $\Delta \overline{l_{b}}$ are used on the body surface and on the free surface within a prescribed radius from the axis of symmetry. Beyond the prescribed radius, element size increases gradually until it reaches a maximum. The prescribed radius on the free surface is taken as $5 \bar{a}$ and the control surface is truncated at $50 \bar{a}$ as a cylinder in this paper. As described in section 2.1, the critical distance $\Delta \bar{l}_{c}$ for the free-surface breakup and the flow detachment is taken as $10 \%$ of $\Delta \bar{l}_{b}$. This will become smaller when $\Delta \bar{l}_{b}$ decreases and therefore the sensitivity of the effect of $\Delta \bar{l}_{c}$ is embedded in the convergence study with element size.

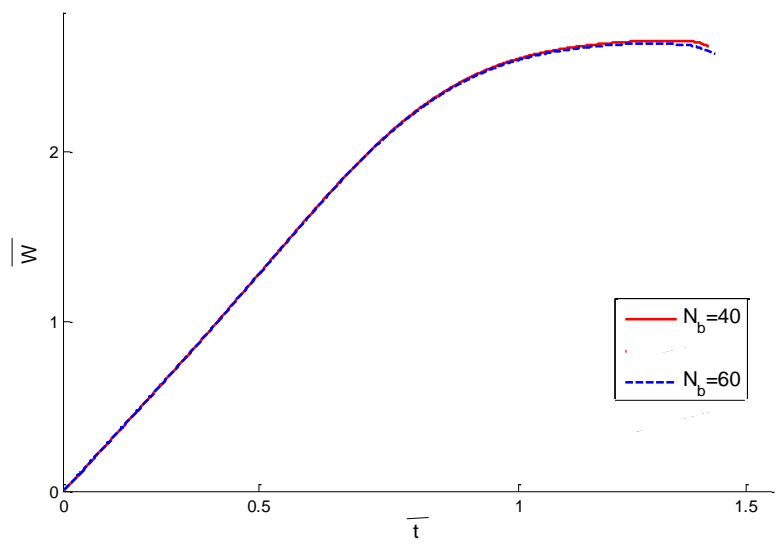

(a)

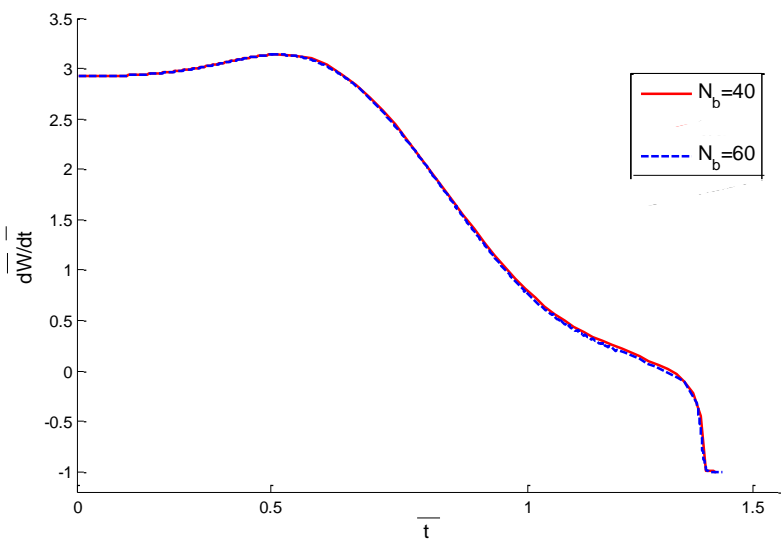

(b)

Fig. 2 Convergence study with element size, the time history of (a) velocity $W$ and (b) acceleration $d W / d t$ of 
the body

Fig.2 gives the time histories of the velocity and acceleration of the body with two different meshes, until the moment of the flow detachment. The figure shows good mesh convergence. At a smaller $\Delta \bar{l}_{c}$ the time of detachment is slightly delayed. This is understandable based on the present methodology, as the body will move a bit further up and the wetted surface at the bottom of the body will become a bit smaller before the liquid detachment in the numerical simulation. It is interesting to see that during this period of delayed detachment the nondimensionlized acceleration of the body is almost -1 . This indicates that the body is virtually in the free fall motion and the hydrodynamic force from the very small wetted surface is negligible. Thus a small delay of the liquid detachment does not affect the real physics of interest.

\subsubsection{Comparison study}

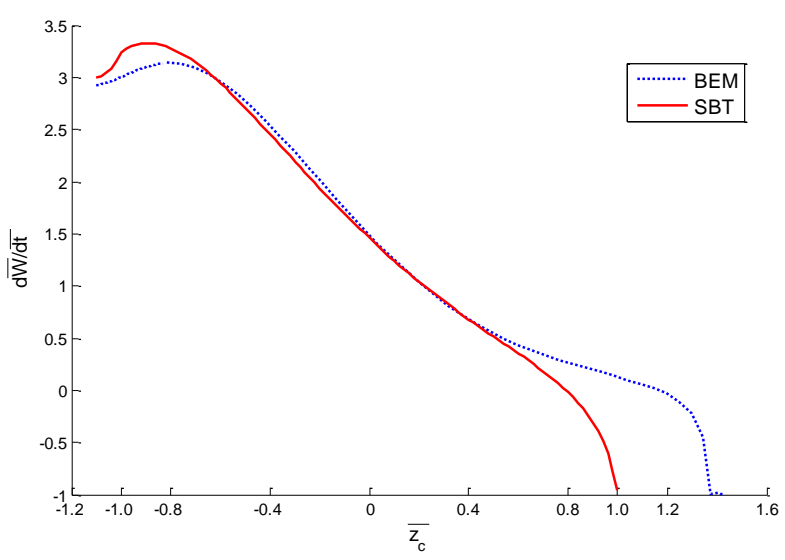

Fig. 3. Acceleration of the body as a function of $\bar{z}_{c}$

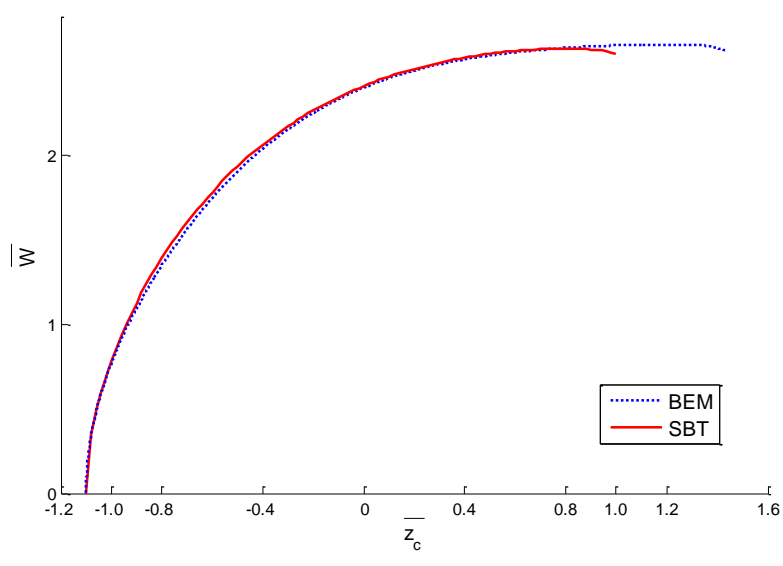

Fig. 4. Velocity of the body as a function of $\bar{z}_{c}$

We then compare the numerical results of BEM with those from the slender body theory (SBT).

The initial total number of elements on the body surface in the meridian plane is $N_{b}=40$, and value of other parameters are taken as the same as those in section 3.1.1. Fig.3 presents the variation of acceleration of the body as a function of $\bar{z}_{c}$, by using SBT and BEM respectively. It can be seen the results from these two methods agree well, especially in the middle part of the 
curve which corresponds to post breakup of the free surface. In SBT, the calculation terminates at $\bar{z}_{c}=1$ when the body departs from the flat free surface. BEM simulation continues because of the rise of the free surface. This is the principal reason why these two curves have a bigger difference at the final stage of water exit. It can be seen that $d \bar{W} / d \bar{t}=-1$ when the body detaches from the water, as the fluid force becomes zero and the external force is gravity only. Fig. 4 provides the variation of velocity of the body with its position. The agreement between results from SBT and BEM is even better than that in Fig.3.

To further check the accuracy of BEM results, an equation alternative to Eq.(21) is used to obtain the force. We may use (Wu \& Ma, 1995):

$$
\bar{F}_{z}=-\frac{d}{d \bar{t}} \iint_{\bar{s}_{b}(\bar{t})} \bar{\Phi} n_{z} d \bar{s}+\iint_{\bar{s}_{b}(\bar{t})}\left[\frac{\partial \bar{\Phi}}{\partial \bar{z}} \frac{\partial \bar{\Phi}}{\partial n}-\frac{1}{2}(\nabla \bar{\Phi})^{2} n_{z}\right] d \bar{s}-\iint_{\overline{s_{b}}(\bar{t})} \bar{z} n_{z} d \bar{s}+\int_{\bar{c}(\bar{t})} \bar{\Phi} \frac{\partial \bar{\Phi}}{\partial \bar{r}} d \bar{c},
$$

where $\bar{c}(\bar{t})$ is the waterline of the body. The last term on the right hand side of Eq.(37) is zero before body pierces the free surface. The first term is calculated using the difference method. This equation is usually accurate, apart from at the time step when remeshing is applied. In such a case the difference due to different meshes at $\bar{t}$ and $\bar{t}+\Delta \bar{t}$ may be amplified by the first term. Fig.5 gives the results from the two methods. It can be seen that the forces from Eqs. (21) and (37) agree well both before and after the free-surface breakup.

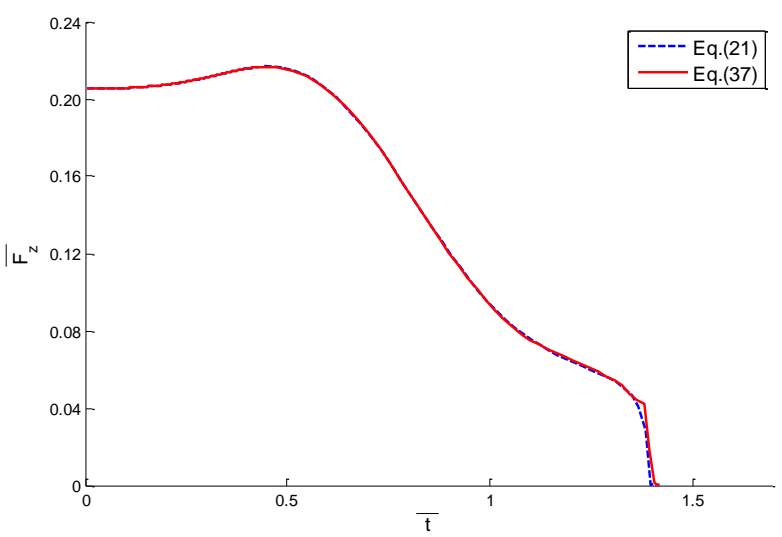

Fig. 5. Time history of the fluid force on the body 
We may also check energy conservation in our results. The total mechanical energy $\bar{E}_{f}(\bar{t})$ of the flow, including the kinetic and gravitational potential energies, can be written as

$$
\bar{E}_{f}(\bar{t})=\iint_{\bar{V}(\bar{t})}\left(\frac{1}{2} \nabla \bar{\Phi} \nabla \bar{\Phi}+\bar{z}\right) d \bar{v}=\frac{1}{2} \iint_{\bar{s}(t)}\left(\bar{\Phi} \frac{\partial \bar{\Phi}}{\partial n}+\bar{z}^{2} n_{z}\right) d \bar{s}=\frac{1}{2} \iint_{\bar{s}_{b}+\bar{s}_{f}} \bar{\Phi} \frac{\partial \bar{\Phi}}{\partial n} d \bar{s}+\frac{1}{2} \int_{\bar{s}_{b}+\bar{s}_{f}+\int_{\bar{s}}} \bar{z}^{2} n_{z} d \bar{s}
$$

The energy $\bar{E}_{b}(\bar{t})$ of the body is

$$
\bar{E}_{b}(\bar{t})=\frac{1}{2} \bar{m} \bar{W}^{2}+\bar{m} \bar{z}_{c}
$$

From the energy conservation law, we should have $\Delta \bar{E}=\bar{E}(\bar{t})-\bar{E}(0)=0$, where $\bar{E}(\bar{t})=\bar{E}_{f}(\bar{t})+\bar{E}_{b}(\bar{t})$.

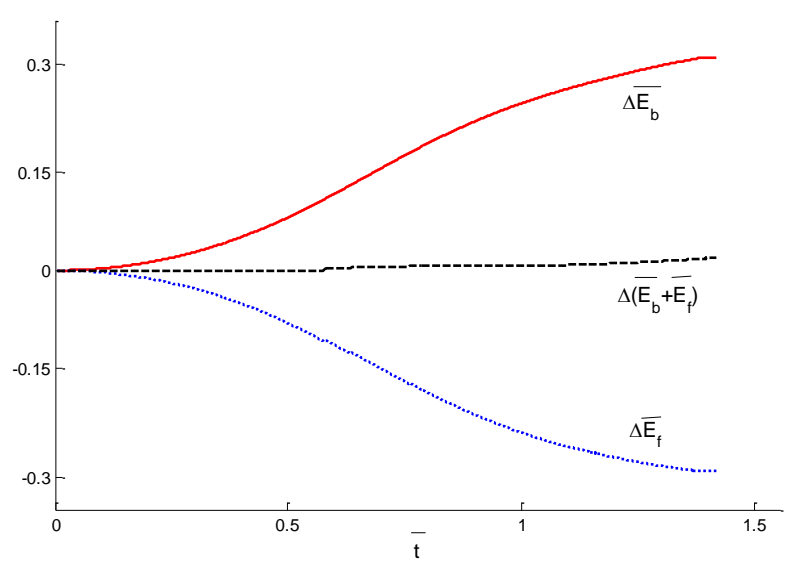

Fig. 6. Time histories of mechanical energy variations of the fluid and the body

Fig.6 gives the time histories of energy variations relative to their corresponding initial values of the fluid and the body. It can be seen that $\Delta \bar{E}$ is almost zero, which is in fact $\Delta \bar{E}<2 \times 10^{-4}$ in our numerical calculation, in the entire simulation and it shows that the energy conservation has been maintained well. 


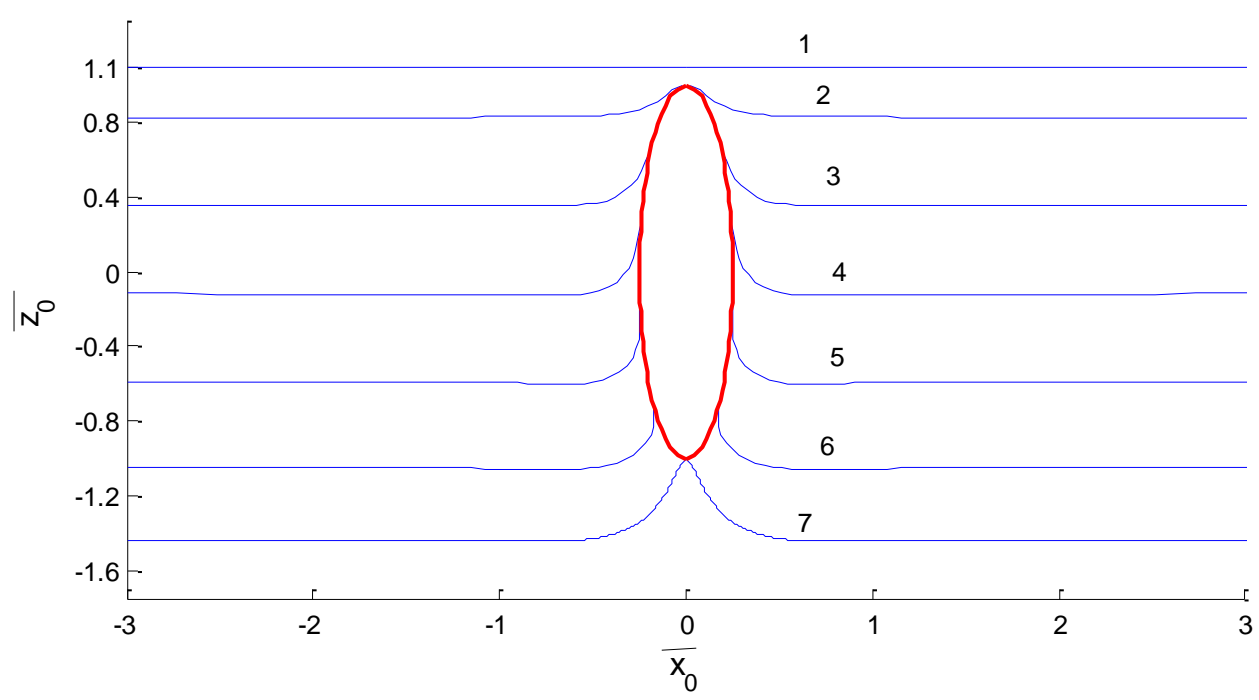

Fig.7 Free-surface profiles in the $\bar{y}_{0}=0$ plane in the moving coordinate system (1): $\bar{t}=0,(2)$ : $\bar{t} \approx 0.4305,(3): \bar{t} \approx 0.7088,(4): \bar{t} \approx 0.9142,(5): \bar{t} \approx 1.0977,(6): \bar{t} \approx 1.2580$, and (7): $\bar{t} \approx 1.4191$.

After the convergence study and comparison, Fig. 7 provides the free surface at $\bar{y}_{0}=0$ plane in the moving coordinate system $O-\bar{x}_{0} \bar{y}_{0} \bar{z}_{0}$ with its origin fixed at the centre of the prolate ellipsoid. The initially stationary body accelerates suddenly under the action of its net vertical force and pushes the water right above upwards and sideway. When the body is close to the free surface, the water layer above the top of the body becomes thinner and thinner. In Fig.7 (2), the very thin water layer has just been removed based on the numerical procedure outlined in Section 2.1 and the body has just pierced the free surface. After that the body continues moving upwards, while the waterline, or the intersection of the free surface and the body surface, stays above the undisturbed free surface. The water detaches from the body at $\bar{t} \approx 1.4191$ in Fig.7 (7). It can be seen a water column below the body has formed at the time of detachment and the free surface is expected to oscillate afterwards under the gravitation. The disturbance will be propagated away in the form of wave radiation.

\subsection{Case study}


We now further undertake three sets of case studies corresponding to the variations of body density $\bar{\rho}_{B}$, minor axis $\bar{a}$ and initial submergence parameter $\lambda$. Elements discretization is the same as that in section 3.1.2 with $N_{b}=40$.

\subsubsection{Body density $\bar{\rho}_{B}$}

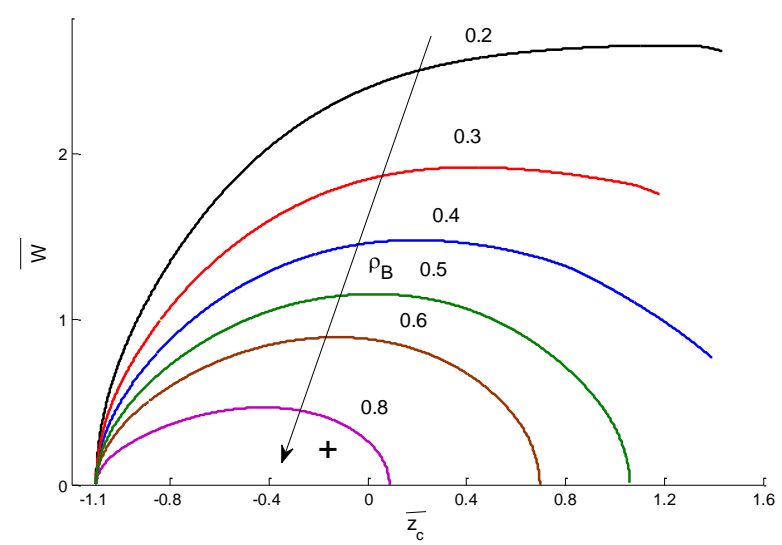

Fig. 8. Velocity of the body as a function of $\bar{z}_{c}$ at different body density $(\bar{a}=1 / 4$ and $\lambda=1.1$, simulation terminates at the moment of either $\bar{W}=0$ or the body detaching from water)

As can be expected, body density $\bar{\rho}_{B}$ is a dominant parameter in the free water exit of a buoyant body. We take $\bar{a}=1 / 4, \lambda=1.1$ and change $\bar{\rho}_{B}$ from 0.1 to 0.9 with interval 0.1 . Fig. 8 provides the velocity of the body as a function of $\bar{z}_{c}$ at different body density, where the arrow indicates the direction of increasing $\bar{\rho}_{B}$. Based on Eq.(36) of SBT, the critical density for complete water exit is $\bar{\rho}_{B, c}=11 / 21 \approx 0.5238$. In the BEM, the critical density can be established based on the assumption that there will be full water exit if when the body speed $\bar{W}=0$, the difference between its acceleration $d \bar{W} / d t$ and that due to gravity is less than $1 \%$. This condition in fact implies that there is hardly any water attached on the body as there is hardly any fluid force. Based on this, our numerical tests have shown that when $\bar{\rho}_{B}=0.2,0.3$ or 0.4 the body exits water fully, while at $\bar{\rho}_{B}=0.5,0.6$ or 0.8 the body will emerge from water but will not detach 
from the water, or it is a partial water exit. Thus the critical body density $0.4<\bar{\rho}_{B, c}<0.5$, which is lower than that from SBT with a flat surface. There are obviously two reasons for this. Firstly in the fully nonlinear theory, some of the body energy will be converted into the potential energy of the fluid as well as its kinetic energy. Thus, there may be less energy in the body to maintain its rise and the body has to be lighter if it is to detach water completely. The second reason is that in SBT, the body is out of water fully when $\bar{z}_{c} \geq 1$ or when the lowest point of the body is above the flat free surface. In the fully nonlinear BEM, the water surface moves up and the lowest point of the body is still in the water at $\bar{z}_{c}=1$. From Fig. 8 , one can see when a body detaches from water, $\bar{z}_{c}>1$.

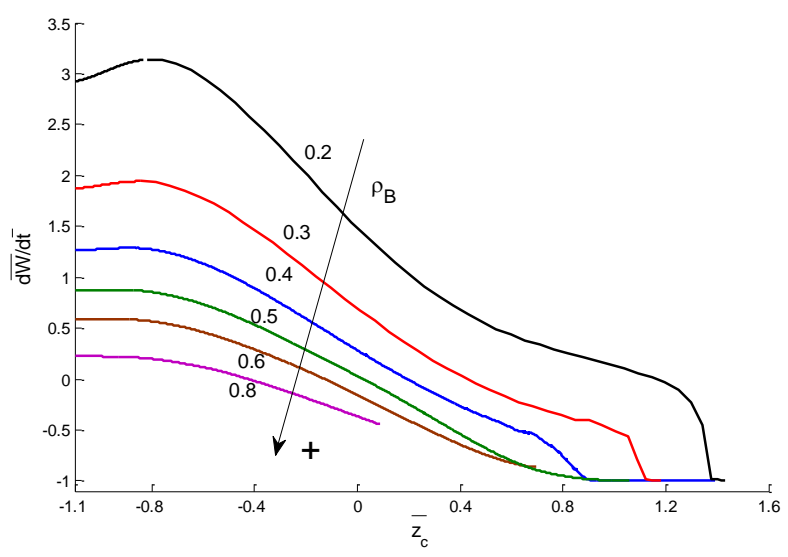

Fig. 9. Acceleration of the body as a function of $\bar{z}_{c}$ at different body density $(\bar{a}=1 / 4$ and $\lambda=1.1)$

Fig. 9 provides the acceleration as a function of $\bar{z}_{c}$. It can be seen the lighter the body is, the larger the acceleration is. This is very much due to the larger difference between the buoyancy and weight, which acts on a body of smaller mass. Furthermore, the dynamic effect becomes more significant for the lighter body, through which the acceleration of the body rises before it falls. For the cases of body existing water fully, the acceleration of the body with small density (such as 0.2 , 0.3) falls sharply to -1 just before departing from the water, which means a rapid decline of hydrodynamic force. While for the one with density close to the critical density $\bar{\rho}_{B, c}$ (such as $\bar{\rho}_{B}$ 
$=0.4$ ), the acceleration falls more gently to -1 . In fact there is a relatively long period over which the acceleration is close to -1 before the body detaches from water. This is because the velocity of the body is close to zero at the final stage when $\bar{\rho}_{B}$ is close to the critical value. A small wetted surface will remain for a longer period of time, over which the fluid force is nearly zero.

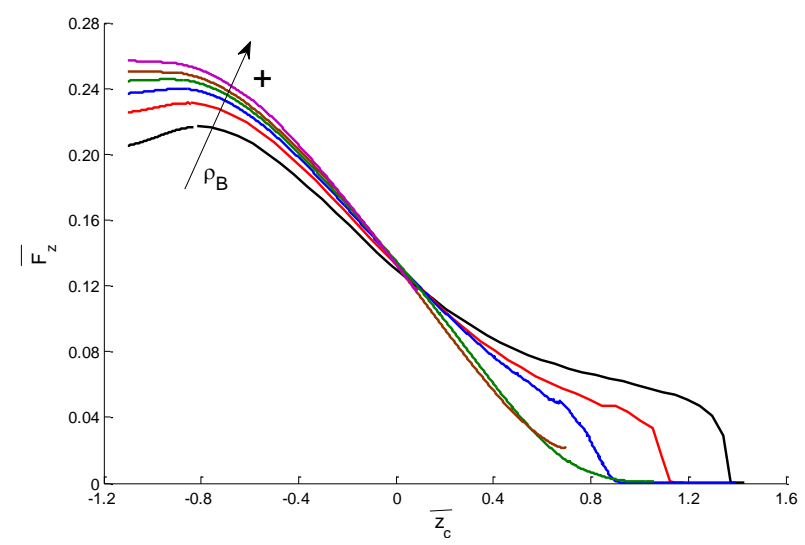

Fig. 10. Fluid force on the body as a function of $\bar{z}_{c}$ at different body density $(\bar{a}=1 / 4$ and $\lambda=1.1)$

Fig.10 gives the fluid force as a function of $\bar{z}_{c}$. It is interesting to see that the force on a heavier body is actually larger than that of a lighter one in the initial stage, although its acceleration is smaller. We may use Eq.(19) at $\bar{t}=0$. This gives

$$
\frac{d \bar{W}}{d \bar{t}}=\frac{\bar{V}_{B}\left(1-\bar{\rho}_{B}\right)}{\bar{\rho}_{B} \bar{V}_{B}+\bar{m}_{a}} .
$$

For a lighter body of smaller $\bar{\rho}_{B}, d \bar{W} / d \bar{t}$ is obviously larger. However from Eqs. (21) and (40), we have at $\bar{t}=0$

$$
\bar{F}_{z}=\frac{\bar{V}_{B}+\bar{m}_{a}}{1+\frac{\bar{m}_{a}}{\bar{\rho}_{B} \bar{V}_{B}}} .
$$

This shows that $\bar{F}_{z}$ is larger for a body of the same shape but bigger $\bar{\rho}_{B}$, which is reflected in 
Fig.10. It is also interesting to see that while the acceleration of the body in Eq.(40) can change sign, depending on whether the body is heavier or lighter than the liquid, the fluid force in Eq.(41) is always upwards. One should note that $\bar{F}_{z}$ is the total fluid force including both hydrodynamic and hydrostatic forces and the latter is always positive.

\subsubsection{Minor semi axis $\bar{a}$}

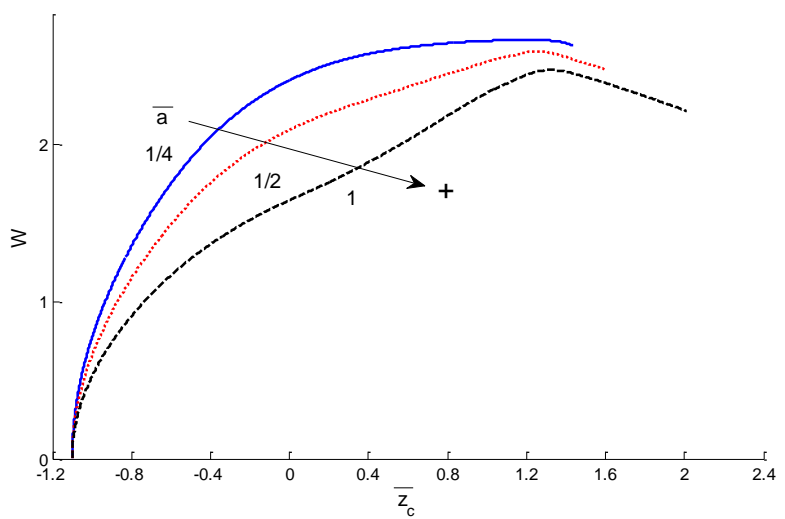

Fig. 11. Velocity of body as a function of $\bar{z}_{c}$ at different $\bar{a}\left(\bar{\rho}_{B}=0.2\right.$ and $\left.\lambda=1.1\right)$

Fig.11 provides the velocity of body with $\bar{\rho}_{B}=0.2$ and $\lambda=1.1$ at different $\bar{a}$. Eq.(36) shows that the critical density does not depend on $\bar{a}$. However, the equation is based on the slender body theory. We have therefore undertaken further numerical tests and found that all these bodies will exit water fully based on the present numerical condition. The simulations in the figure are terminated at the moment of water detachment. It can be seen that a more slender body has a bigger rising velocity, and it will exit water fully at a smaller $\bar{z}_{c}$. On the other hand, the velocity curve of the sphere $(\bar{a}=1)$ has a more distinct peak with larger gradients on both sides, which implies that the acceleration changes from positive to negative more rapidly there. 


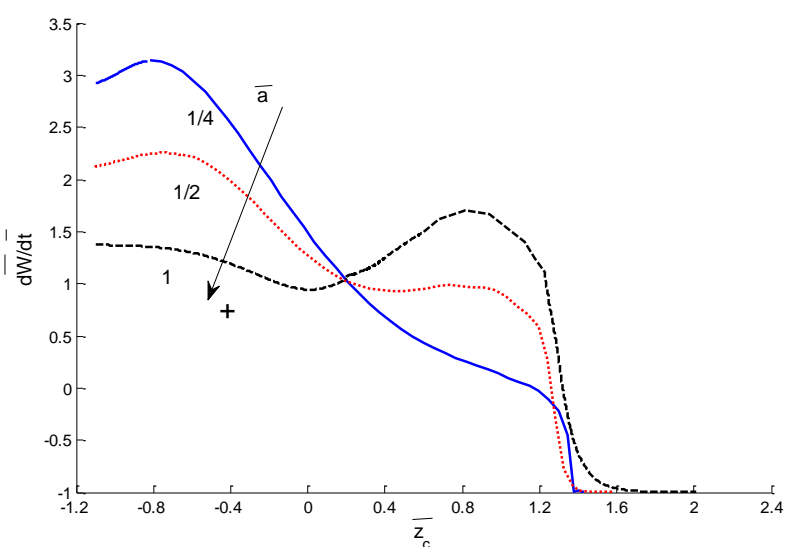

Fig. 12. Acceleration of body as a function of $\bar{z}_{c}$ at different $\bar{a} \quad\left(\bar{\rho}_{B}=0.2\right.$ and $\left.\lambda=1.1\right)$

Fig. 12 provides the acceleration of the body at different $\bar{a}$. It shows that the body with a smaller $\bar{a}$ corresponds to a larger initial acceleration. We notice that the ratio of buoyancy and weight of the body is the same for these bodies. The acceleration will obviously very much depend on the ratio of added mass to the body mass. One can expect that a slender body has a smaller added mass ratio therefore a larger acceleration. In fact from Eq.(40), we have $\frac{d \bar{W}}{d \bar{t}}=\frac{1-\bar{\rho}_{B}}{\bar{\rho}_{B}+k_{s}}$. Because the ratio of the added mass to the body volume $k_{s}$ decreases for a body with a smaller $\bar{a}$ (Lamb, 1932), $d \bar{W} / d \bar{t}$ is larger at smaller $\bar{a}$. For the cases of $\bar{a}=1 / 4$ and $1 / 2$, the acceleration increases with $\bar{z}_{c}$ initially and then decreases all the way. For $\bar{a}=1$ or the sphere, however, it decreases first and then rises. The acceleration rises to a peak near the moment of the body detachment from water and then it drops very rapidly to the acceleration of free fall. This suggests a rapid variation of the fluid force. 


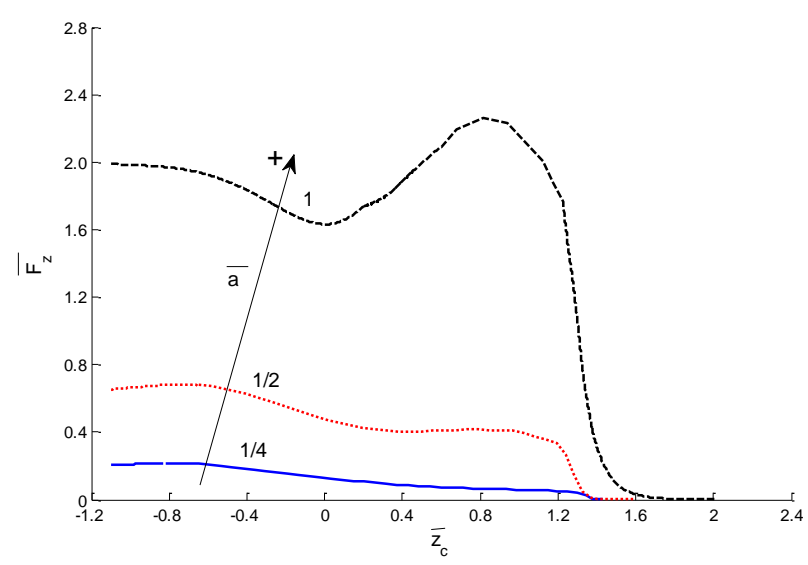

Fig. 13. Fluid force on the body as a function of $\bar{z}_{c}$ at different $\bar{a} \quad\left(\bar{\rho}_{B}=0.2\right.$ and $\left.\lambda=1.1\right)$

Fig. 13 gives the fluid force on the body. It can be seen that a body of larger $\bar{a}$ has a larger fluid force all the time, although its acceleration in Fig.12 is smaller at the initial stage. From Eq.(41), one has $\bar{F}_{z}=\frac{4}{3} \pi \bar{a}^{2} \bar{\rho}_{B}\left(\frac{1-\bar{\rho}_{B}}{\bar{\rho}_{B}+k_{s}}+1\right)$ at $\bar{t}=0 . \quad k_{s}$ increases with $\bar{a}$ at lower rate than $\bar{a}^{2}$ (Lamb, 1932) and therefore $\bar{F}_{z}$ increases with $\bar{a}$. We notice that the fluid force on the sphere $(\bar{a}=1)$ reaches a maximum and then drops rapidly before the body departs from water. To get some insight, we further plot the free-surface profiles for body of $\bar{a}=1$ in the $\bar{y}_{0}=0$ plane in the moving coordinate system in fig.14. We notice that in contrast to Fig.7, the free surface for the sphere, a non-slender body, has a long and thin water layer attached on the body surface after body nose exits water, which is in the form of a two dimensional jet in each given azimuth. As the sphere continues to move upwards, the jet is sliding along the body surface and its length is reducing. However, the curvature at jet root is getting quite large as profiles 4-5 in Fig. 4 shows. The large curvature usually corresponds to a large pressure gradient and large pressure itself for the reason discussed by Wu \& Sun (2014). When the jet root moves towards the bottom of the sphere, it makes a large contribution to the vertical direction, and therefore a rise of the vertical force in Fig.13 and the acceleration in Fig.12. The force drops to zero and the acceleration becomes -1 when the body detaches from water. 


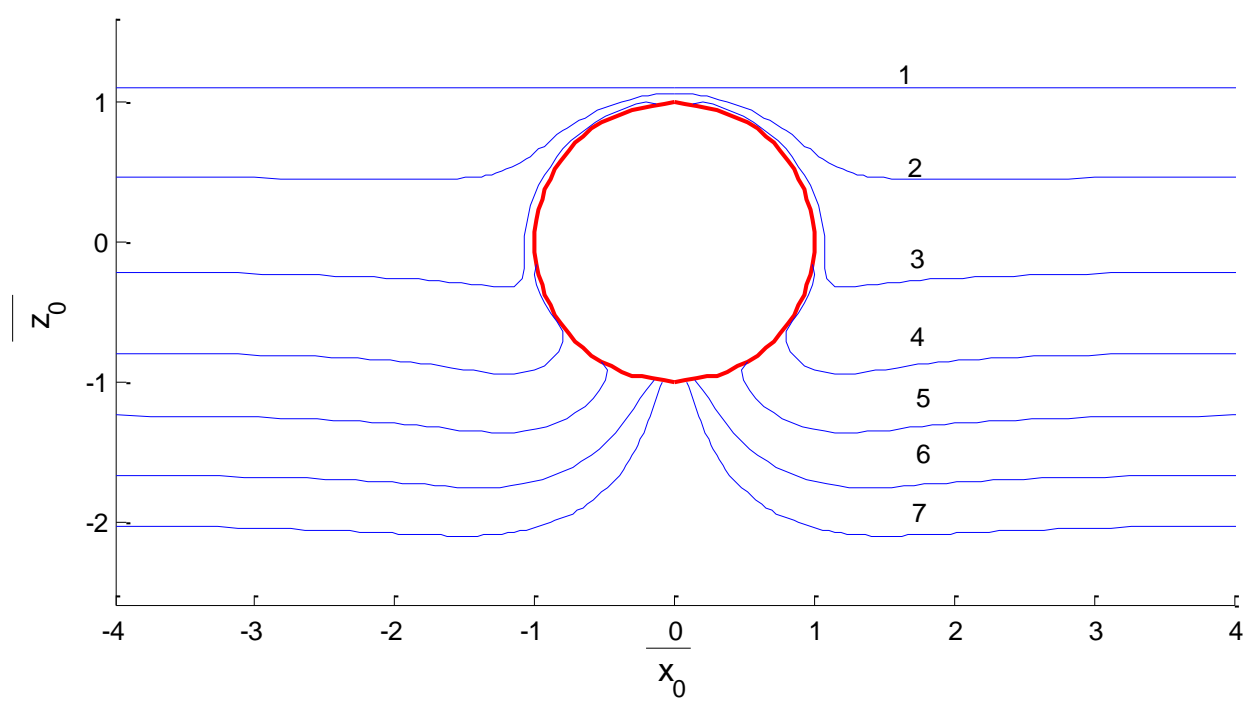

Fig. 14 Free-surface profiles for a sphere in the $\bar{y}_{0}=0$ plane in the moving coordinate system (1): $\bar{t}=0, \quad \bar{z}_{c}=-1.1,(2): \bar{t} \approx 0.9621, \quad \bar{z}_{c} \approx-0.4708,(3): \bar{t} \approx 1.4001, \quad \bar{z}_{c} \approx 0.2010,(4): \bar{t} \approx 1.7138$, $\bar{z}_{c} \approx 0.8134,(5): \bar{t} \approx 1.8891, \quad \bar{z}_{c} \approx 1.2212,(6): \bar{t} \approx 2.0619, \quad \bar{z}_{c} \approx 1.6426$, and $(7): \bar{t} \approx 2.2222$, $\bar{z}_{c} \approx 2.0100\left(\bar{a}=1, \bar{\rho}_{B}=0.2\right.$ and $\left.\lambda=1.1\right)$.

3.2.3 Initial submergence parameter $\lambda$

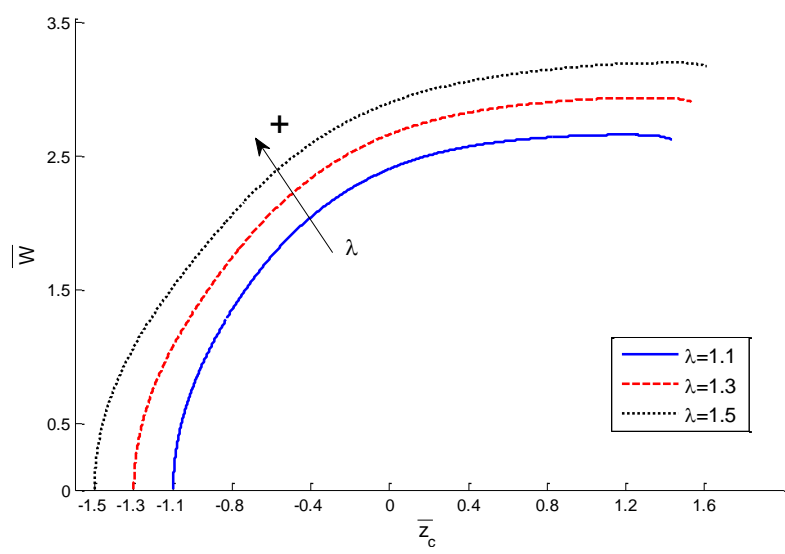

Fig. 15. Velocity of body as a function of $\bar{z}_{c}$ at different initial submergence parameter ( $\bar{\rho}_{B}=0.2$ and $\bar{a}=1 / 4)$

Fig. 15 presents velocity of body of $\bar{\rho}_{B}=0.2$ and $\bar{a}=1 / 4$ as a function of $\bar{z}_{c}$ at different initial submergence parameters, $\lambda=1.1,1.3$ and 1.5 . The body exits water fully in all the cases. 
It can be seen that the velocity increases as $\lambda$ gets larger at a given $\bar{z}_{c}$. This is understandable because the lower point is, from which the light body starts to move, the larger velocity it can reach at the same $\bar{z}_{c}$. Based on Eq.(36), this also makes it easier for the body to exit water fully.

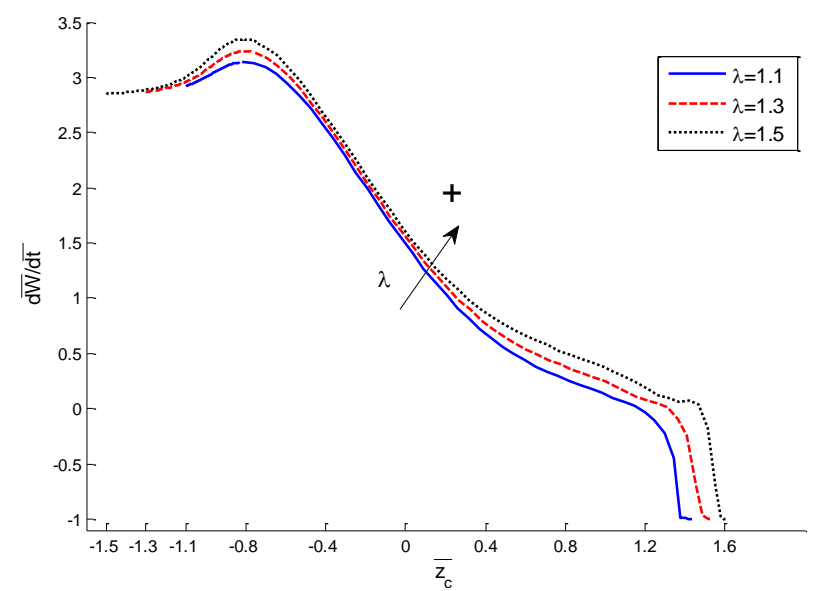

Fig. 16. Acceleration of body as a function of $\bar{z}_{c}$ at different initial submergence parameter $\left(\bar{\rho}_{B}=0.2\right.$ and $\left.\bar{a}=1 / 4\right)$

Fig.16 provides acceleration as a function of $\bar{z}_{c}$. All the curves of acceleration have similar trends. A large part of these curves are very close to each other. This implies the dominant force may be buoyancy of this body. Overall the effect of $\lambda$ on the acceleration is insignificant. The curve for $\bar{F}_{z}$ is the same as that for $d \bar{W} / d \bar{t}$, because the body mass $\bar{m}$ is the same in all these cases.

\section{Conclusions}

Water exit of a fully-submerged buoyant body in free motion is simulated by using the boundary-element method for the velocity potential flow theory with the fully nonlinear boundary conditions. Extensive results for spheroids have been obtained, from which the following conclusions can be drawn. 
(1) The body density is a dominant factor during free water exit. There exists a critical value which divides full and partial water exit. This value has been found as $\bar{\rho}_{B, c}=\frac{h}{h+b}$ for a spheroid of initial submergence $h$ and semi major axis $b$, based on the slender body theory with the assumption of zero potential on the flat free surface. This critical value varies with $h$ for a given body and is within the range of $\frac{1}{2}<\bar{\rho}_{B, c}<1$, as $h>b$. A larger initial submergence $h$ gives a larger $\bar{\rho}_{B, c}$, which allows a heavier body to exit water completely.

(2) During the entire water exit, a lighter body always has a larger acceleration than the heavier one. This is because they have the same buoyancy and same added mass. A lighter body gives a smaller resistance to the body, and therefore it has a larger net force and a smaller total mass term. The fluid force at the initial stage is due to the difference between the buoyancy force and the inertial force. As the heavier body has a smaller acceleration while its added mass is the same as the lighter body, it has a larger net fluid force.

(3) At the same density, a more slender body always has a larger velocity during water exit. For a blunter body, namely a sphere in the present case, the force and acceleration will have a peak right before the body detaches from water. This is caused by the large pressure at the jet root associated with a blunter body. As the jet root moves towards the blunter bottom before detaching from the body, it makes a larger contribution to the vertical force.

\section{Acknowledgements}

This work is supported by the National Natural Science Foundation of China (Nos. 51639004, 11472088, and 51579054), the International Postdoctoral Exchange Fellowship Program (No. 20140068) to the first author and hosted by University College London, and Lloyd's Register Foundation through the joint centre involving University College London, Shanghai Jiaotong University and Harbin Engineering University, to which the authors are most grateful. Lloyd's Register Foundation helps to protect life and property by supporting engineering-related education, public engagement and the application of research. 


\section{References}

[1]. Baarholm, R., Faltinsen, O.M., 2004. Wave impact underneath horizontal decks. Journal of Marine Science and Technology 9, 1-13.

[2]. Bourrier, P., Guyon, E., Jorre, J. P., 1984. The 'pop off' effect: different regimes of a light ball in water. European Journal of Physics 5(4), 225-231. (in French)

[3]. Greenhow, M., Moyo, S., 1997. Water entry and exit of horizontal circular cylinders. Philosophical Transactions of the Royal Society A 355, 551-563.

[4]. Khabakhpasheva, T.I., Korobkin, A.A., Mari, K.J., 2015. A linearized exit model for prediction of forces on a body within the $2 \mathrm{D}+\mathrm{T}$ framework. In: $30^{\text {th }}$ International Workshop on Water Waves and Floating Bodies. April 12-15, Bristol, UK.

[5]. Korobkin, A.A., 2013. A linearized model of water exit, Journal of Fluid Mechanics 737, 368-386.

[6]. Korobkin, A.A., Khabakhpasheva, T.I., Mari, K.J., 2014. Water-exit problem with prescribed motion of a symmetric body. In: $29^{\text {th }}$ International Workshop on Water Waves and Floating Bodies. March 30-April 2, Osaka, Japan.

[7]. Lamb, H. Hydrodynamics, 1932, sixth ed. Cambridge University Press.

[8]. Liju, P.Y., Machane, R., Cartellier, A., 2001. Surge effect during the water exit of an axis-symmetric body traveling normal to a plane interface: experiments and BEM simulation. Experiments in Fluids 31: 241-248.

[9]. Longuet-higgins, M. S., Cokelet, E. D., 1976. The deformation of steep surface waves on water. I. A numerical method of computation. Proceedings of the Royal Society A, 350: 1-26.

[10].Mackie, A. G., 1962. A linearized theory of the water entry problem. The Quarterly Journal of Mechanics and Applied Mathematics, 15(2), 137-151.

[11].Moore, M.R., Howison, S.D., Ockendon, J.R., Oliver, J.M., 2012. Three-dimensional oblique water-entry problems at small deadrise angles. Journal of Fluid Mechanics 711, 259-280.

[12].Moran, J. P., 1963. Line source distributions and slender-body theory. Journal of Fluid Mechanics, 17(02), 285-304. 
[13]. Moran, J. P., 1964. Image solution for vertical motion of a point source towards a free surface. Journal of Fluid Mechanics, 18(02), 315-320.

[14].Moran, J. P., 1965. On the hydrodynamic theory of water-exit and-entry. Therm Advanced Research Inc Ithaca NY.

[15].Ni, B.Y., Zhang, A.M., Wu, G.X., 2013. Numerical simulation of motion and deformation of ring bubble along body surface, Applied Mathematics and Mechanics-English edition, 34(12), 1495-1512.

[16].Ni, B.Y., Zhang, A.M., Wu, G.X., 2015. Simulation of complete water exit of a fully-submerged body, Journal of Fluids and Structures, 58,79-98.

[17]. Newman, J. N., 1977. Marine hydrodynamics. MIT press.

[18].Pansiot, J., Lo, B., Yang, G. Z., 2010. Swimming stroke kinematic analysis with BSN. In Body Sensor Networks, 2010 International Conference on IEEE, June

[19].Rajavaheinthan, R., Greenhow, M., 2015. Constant acceleration exit of two-dimensional free-surface-piercing bodies, Applied Ocean Research, 50,30-46.

[20]. Scolan, Y.M., Korobkin, A.A., 2001. Three-dimensional theory of water impact, Part 1: Inverse Wagner problem, Journal of Fluid Mechanics 440, 293-326.

[21].Tassin, A., Piro, D.J., Korobkin, A.A., Maki, K.J., Cooker, M.J., 2013. Two-dimensional water entry and exit of a body whose shape varies in time. Journal of Fluids and Structures 40, $317-336$.

[22].Truscott, T. T., Epps, B. P.,Munns, R. H., 2016. Water exit dynamics of buoyant spheres, Physical Review Fluids, 1(7), 074501.

[23]. Tyvand, P. A., Miloh, T., 1995. Free-surface flow due to impulsive motion of a submerged circular cylinder. Journal of Fluid Mechanics 286, 67-101.

[24].Wu, G.X., 1998. Hydrodynamic force on a rigid body during impact with liquid. Journal of Fluids and Structures 12: 549-559.

[25].Wu, G.X., Eatock Taylor, R. 2003. The coupled finite element and boundary element analysis of nonlinear interactions between waves and bodies. Ocean Engineering 30, 387-400 
[26].Wu, G.X., Ma, Q.W., 1995. Finite element analysis of nonlinear interactions of transient waves with a cylinder. In: $13^{\text {th }}$ International Conference on Ocean, Offshore and Arctic Engineering, Copenhagen, Denmark

[27].Wu G. X., Sun S. L. 2014. Similarity solution for oblique water entry of an expanding paraboloid. Journal of Fluid Mechanics, 745: 398-408.

[28].Wu, G.X., Sun, H, He, Y.S. 2004. Numerical simulation and experimental study of water entry of a wedge in free fall motion. Journal of Fluids and Structures, 19, 277-289. 